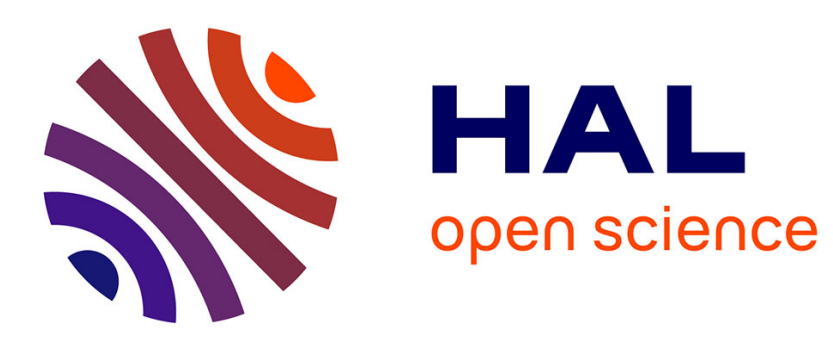

\title{
Das Recht - Ein Territorium staatlicher Hoheit?
}

\author{
Antoine Pelicand
}

\section{To cite this version:}

Antoine Pelicand. Das Recht - Ein Territorium staatlicher Hoheit?: Die Einführung "bürgernaher

Richter" in Frankreich. Zeitschrift für Rechtssoziologie, 2006, 27 (2), pp.263-273. halshs-00128800

\section{HAL Id: halshs-00128800 \\ https://shs.hal.science/halshs-00128800}

Submitted on 2 Feb 2009

HAL is a multi-disciplinary open access archive for the deposit and dissemination of scientific research documents, whether they are published or not. The documents may come from teaching and research institutions in France or abroad, or from public or private research centers.
L'archive ouverte pluridisciplinaire HAL, est destinée au dépôt et à la diffusion de documents scientifiques de niveau recherche, publiés ou non, émanant des établissements d'enseignement et de recherche français ou étrangers, des laboratoires publics ou privés. 


\title{
Das Recht - ein Territorium staatlicher Hoheit?
}

\author{
Die Einführung „bürgernaher“ Richter in Frankreich.
}

\author{
Antoine Pelicand
}

Zusammenfassung: Seit 2002 gibt es eine neue Kategorie von Richtern im franæösischen Gerichtswesen: die „bürgernabe" Richter. Ihr Auftrag ist es, ein anderes Modell der Justiz qu fördern, bei dem die Schlicbtung und das Informelle im Mittelpunkt stehen. Der Artikel stellt diese Reform in die Perspektive der Veränderungen von Staatsregulierung dar. Wenn man die Vereinheitlichung des Justiz̨yesens am Ende des 19. Jabrbunderts qum Vergleich herangieht, wird deutlich, daß die Professionalisierung des Richteramtes und der wachsende Rekurs auf das Gesetz für die Konfliktlösung mit der qunehmenden Regulierung der Gesellschaft seitens eines jakobinischen Staates verbunden ist. Trotzdem erlaubt die heutige Infragestellung der Staatsrolle keine einfache Einführung alternativer Methoden der Streitschlichtung im Gerichtswesen.

Summary: The year 2002 witnessed the creation of a new variety of judge in France. Referred to as "proximity judges", their introduction to the French justice system attempted to build an alternative justice founded upon conciliation and informal procedures. The article examines court reforms from the perspective of changing state regulation. A comparison with the unification of civil and criminal justice at the beginning of the twentieth century indicates that judges' increasing professionalism and the growing tendency to resort to law for dispute resolution are related to an expanding involvement of the Jacobin state in social regulation. Nevertheless, the current destabilization of the state does not allow much further development of alternative solutions.

Der Ausgangspunkt der hier ausgeführten Überlegungen sind die aktuellen Entwicklungen der alternativen Streitbeilegung, angesichts derer die bisherigen Erklärungen oftmals unzureichend sind. Die Argumentation bezieht sich häufig auf gewisse gesellschaftliche Veränderungen (Bonafé-Schmitt 2005). Dem Gedanken der Postmodernität verpflichtet, bestehen zahlreiche Kommentatoren auf der heutigen Entstehung einer neuen Form der sozialen Verhältnisse: einer stärkeren Dezentralisierung der Verantwortlichkeiten, einer höheren Flexibilisierung, einem geringeren Formalismus sowie einer verbesserten Anpassungskapazität. Indem der Begriff der interessengeleiteten Vereinbarung zwischen den betroffenen Individuen, die nunmehr sorgsam auf die Konfliktlösung durch Verhandlung achten, betont wird, stützt sich das Bild der Alternative auf die Dichotomie zwischen dem Öffentlichen und dem Privaten: Es handelt sich insofern um eine Alternative zu dem Öffentlichen, als dass jenes, welches offiziell, zentralisiert und institutionalisiert ist, zugunsten des Informellen verworfen wird, also zugunsten der Flexibilität einer Verhandlung und einer Einigung (Giraud 1993). In vielerlei Hinsicht werden diese Entwicklungen also als ein Prozess der Zivilisierung präsentiert, in dem die Justiz zunehmend einem allgemeinen freien Handel zwischen souveränen Individuen ähnelt (Le Roy 1995).

Tatsächlich muss jedoch festgestellt werden, dass bezüglich der Diskussion um verschiedene Rechtsformen die Unterscheidung zwischen Öffentlichkeit und Privatem im historischen Konzept des Staates begründet ist. Das Private kann als alternative Regelungsinstanz von Streitigkeiten nur als Flucht aus den Regeln des Kollektivs verstanden 
werden, die von der Staatsgewalt aufgezwungen werden. Diese Staatsgewalt wird damit beschuldigt, die Individuen mittels einer kontraproduktiven Prozedur und Technik zu ersticken (Bonafé-Schmitt 1998). Christine B. Harrington hat so bereits betont, wie sehr diese Bewegung der ,alternativen“ Streitschlichtung sich in eine Kultur des Liberalismus einschreibt, die sich auf den Begriff des Marktes und der Interessenskonfrontation sowie des „Minimalstaates“ stützt (Harrington 1988).

Meine Fragestellung stützt sich auf bestimmte Beobachtungen. Die französische Justiz ist heute von einer tiefgehenden Reflexion über ihre Art der Gerichtsbarkeit geprägt. Seit dem Ende der achtziger Jahre ist die Institution das Ziel weitreichend Kritik (Arthuis/Haenel, 1992), die bis zur Frage nach einer Krise des Richtertums geht (Cointat, 2002). Aus diesem Grunde werden seit etwa 15 Jahren mehrere Reformen bezüglich einer Annäherung der Justiz an die Bürger diskutiert. Diese Kritikpunkte betreffen unter anderem die zugrunde liegenden normativen Prinzipien, darunter der öffentliche Charakter des Gerichtsurteils.

Die politisch bevorzugte Variante ist diejenige einer Vereinfachung der Prozeduren und der juristischen Praxis. Einerseits wird so der Zugang zu Gerichten erleichtert (Anruf des Gerichts mittels einer einfachen schriftlichen Erklärung, Senken der Gerichtskosten, ein einziges Sekretariat), andererseits werden die Grenzen des juristischen Systems erweitert, indem konfliktpräventive Regelungen einbezogen werden, neue Akteure (Freiwillige, nicht-professionelle Personen, Vereine) eingeladen werden und alternative Verhandlungsmethoden zusätzlich zu den gesetzlichen erlaubt werden. Vor allem letztere Orientierung kann im Sinne einer Neuorganisation der Justiz in einen öffentlichen und einen privaten Sektor analysiert werden: die außergerichtliche Lösungsmöglichkeiten werden befürwortet, es werden Verantwortliche beauftragt, die nicht zum Öffentlichen Dienst gehören, und das Argumentationsmonopol des Gesetzes wird in Frage gestellt.

Eine Analyse des Falls der französischen Justiz erlaubt ein tiefer gehendes Verständnis der aktuellen Bewegung der „Privatisierung“ der Streitschlichtung. Weshalb will man heute den öffentlichen Charakter der juristischen Arbeit in Frage stellen, indem ein „Schlichtungsbüro" dem Gerichtssaal vorgezogen wird und einem oft mündlichen Abkommen ${ }^{1}$ mehr Wert als einem offiziellen Urteil verliehen wird? Diese Entwicklung in Frankreich hat nichts Naturgegebenes. Das Justizwesen hätte auch seine personellen und materiellen Mittel aufstocken können, ohne seine Praxis zu ändern. Braucht man nicht gerade heutzutage vor allem gerichtliche Entscheidungen, wenn viele sich in Frankreich über das Übermaß subjektiver Ansprüche besorgt zeigen, das eine „Amerikanisierung“ (oder eine „Verrechtlichung") der Gesellschaft befürchten lasse? Doch die gewählte Ausrichtung der Entwicklung zeigt viel eher in die entgegengesetzte Richtung. Dies ist das Paradox, welches wir an dieser Stelle versuchen zu erklären.

Die hier vorgetragene Erklärungsthese betont die Rolle der Entstehung und Konstruktion des Nationalstaates für derartige Entwicklungen des Justizwesens. Innerhalb dieses politischen Rahmens wurde die staatliche Politik lange auf die Interventionen der Zentralregierung beschränkt sowie auf die von ihr begründeten Ziele, deren Verwirkli-

\footnotetext{
An dieser Stelle muss präzisiert werden, dass die alternativen Methoden der Streitbeilegung i.A. oftmals keine schriftliche Festhaltung der Verhandlung und ihrer Ergebnisse vorsehen. Die in Frankreich durchgeführten Studien über die Schlichtungsverfahren (,les conciliateurs de justi$\left.\mathrm{ce}^{\mathrm{c}}\right)$ zeigen beispielsweise, dass die schriftliche Formulierung einer Einigung nur in weniger als $30 \%$ der Fälle angefertigt wird. Desdevises, Yvon, Suaud, Charles (Hrsg.) (2001): Conciliateurs et conciliation, Paris, Mission de recherche Droit et Justice, S. 128.
} 
chung zum Gemeinwohl beitragen sollte. Es handelt sich hier darum, die Auswirkungen eines solchen historischen Phänomens auf die Praxis einer Justiz aufzuzeigen, die sich prinzipiell auf Verhandlungsmethoden und Vertraulichkeit stützte. Die Einmischung des jakobinischen Staates in die Regulierung der Gesellschaft hat sich so, mittels des Rechts, durch die Normierung der juristischen Praxen geäußert. Die aktuelle Wiederkehr der Nähe juristischer Institutionen und Praxis zu den ihr Unterworfenen muss daher im Kontext der strukturellen Infragestellung der staatlichen Macht gesehen werden (Commaille 1999). Um diese Zusammenhänge aufzuzeigen, analysieren wir an dieser Stelle zwei Schlüsselmomente und weisen nach, wie zu diesen historischen Zeitpunkten das Nachdenken über den Staat und über die Justiz verbunden waren. Zum einen war dies der Fall, als die Justiz sich Ende des 19. Jahrhunderts vereinheitlicht hat. Zum anderen ist dies heute dort der Fall, wo eine neue Instanz, die „bürgernahe Justiz“ (Justice de proximitê) konstruiert wird.

Um diese Momente zu analysieren, interessieren wir uns vor allem für die Grenzen der juristischen Institution an derjenigen Stelle, an der Ungewissheiten über die Zuständigkeiten Veränderungen erleichtern können. Dies trifft beispielsweise im Fall der sogenannten „Bagatellstreitigkeiten“ $\mathrm{zu}$, die wegen ihrer geringen finanziellen oder moralischen Implikationen so bezeichnet werden. Offensichtlich zählen sie zu denjenigen sozialen oder ökonomischen Problemen, die aus der Funktionsweise der Gesellschaft heraus entstanden sind, und denen sich das juristische System widmen muss. Gleichzeitig unterschieden sie sich von ihr, da die Richter sie oft eher als der privaten Sphäre zugehörig empfinden und selbst an der Zweckmäßigkeit des Rechtes in diesen Fällen zweifeln.

\section{Ende des 19. Jahrhunderts: Die Justiz zur Stunde des Sozialstaates}

\subsection{Die Grenzen des vereinbeitlichenden revolutionären Werkes}

Wie viele andere Institutionen in Frankreich ist auch die Entstehung der Justiz auf die Zeit der Revolution zurückzuführen. Während der französischen Revolution wird so die juristische Praxis, nunmehr als an Gesetzen orientiert (der „Ausdruck des Volkswillens"), neu konzipiert. Und diese Rationalisierung impliziert auch Transparenz in Form der Öffnung der Prozesse für die Öffentlichkeit, die Stützung der Urteile auf legale Grundlagen sowie die methodische Argumentation der Verteidigung durch die Parteien. Der juristische Apparat, so wie er von den „Constituants" (die ersten Abgeordneten nach 1789) in den Gesetzen des 17. und 24. August 1790 entworfen wurde, begrenzt vor allem den Handlungsraum des Richters. Dieser ist fortan dem Gesetzestext unterworfen. Er solle, so der damalige von Montesquieu inspirierte Ausdruck, ein reiner „Mund des Gesetzes“ sein. Aus diesen Gründen ist die Entstehung des juristischen Systems in Frankreich, welches sich auf universelle Bezüge stützt, mit der Entstehung des Nationalstaates eng verbunden. Von diesem Zeitpunkt an sollten alle Bürger von dem Gesetz einheitlich behandelt werden, um eine strikte Gleichheit zu garantieren.

Dennoch wird dieses juristische Ideal nicht als angemessen für die Gesamtheit aller Konflikte betrachtet. Es steht vielmehr einem revolutionären Konzept gegenüber, einem konkurrierenden Prinzip: Ein wichtiges Anliegen der Revolution ist in der Tat der Kampf gegen endlose Gerichtsprozeduren sowie gegen die Komplexität der Justiz. Diesen Mängeln wird die Regulierungsmacht der Gemeinschaft entgegen gesetzt, die durch die Bürger selbst ausgeübt wird (Glick 1983). Es handelt sich dabei um eine rein lokale Konfliktbeilegung, die von einem Teil der „Constituants" stark befürwortet wird. Hieraus ergab sich 
letztendlich die Entscheidung, die Konflikte nach ihrer Natur und dem Ausmaß der Streitigkeit zu unterscheiden. Lediglich durch seine Begründung in der Effizienz kann sich das Recht nicht als einzige Referenz der Gerechtigkeit durchsetzen. Trotz der enormen Bestrebungen der Vereinfachung und der Rationalisierung des Rechtssystems, die durch die Revolutionäre vollzogen wurde, bestehen weiterhin unterschiedliche Formen der Rechtsprechung, die der Staat nicht in gleichem Maße kontrolliert. Gewiss werden die wichtigsten Angelegenheiten durch einen Berufsrichter entschieden, der durch seine juristischen Kompetenzen qualifiziert ist; aber eine ansehnliche Zahl der Streitigkeiten wird lokalen Akteuren anvertraut, die für eine Beruhigung der Situation Sorge tragen, ohne dass es notwendig wäre, an die Regeln der nationalen Gemeinschaft zu appellieren.

Trotz der Schaffung eines Zentralstaates während der Revolution, der auf verschiedene Weise auf die Vereinheitlichung der Normen und Regeln auf dem nationalen Territorium achtete, wurde soziale Regulierung im Laufe des 19. Jh. zugegebenermaßen zu einem großen Teil durch Verhandlungen, Arrangements und lokale Entscheidungen ausgeübt. Der relevanteste Fall diesbezüglich stellt die Handhabung der Berufsbeziehungen dar. Bis zur Verabschiedung des kodifizierten Arbeitsrechts ab 1886 waren die Beziehungen zwischen Vorgesetztem und Arbeitern in der entstehenden Industrie das Ergebnis lokaler Arrangements und einer örtlichen, unabhängigen Interpretation der revolutionären Prinzipien (insbesondere das vom Deputierten Le Chapelier eingebrachtes Gesetz, das 1791 die Zünfte und Korporationen abgeschafft hatte). Dieses wurde damals als das ,gute Recht" (le „,bon droit") bezeichnet (Cottereau 2002).

Der größte Anteil des Soziallebens wurde also einer Gerichtsbarkeit überlassen, die außerhalb des Rechtes und des Formalismus Justiz ausübte. Dies war vor allem bezüglich der so genannten „Friedensrichter" der Fall, die wegen der Reichweite ihrer Kompetenzen und der großen Anzahl von Anliegen, über die sie urteilen, von hoher Bedeutung sind. Gewählt während der Revolution, später von der Exekutive aus den Reihen der Bevölkerung nominiert, sorgten sie für Gerechtigkeit auf dem gesamten Staatgebiet in über 3000 Kantonen. Anfangs als reine Schlichtungsinstanzen geschaffen, wurden ihre Funktionen und Kompetenzen im Laufe des Jahrhunderts stark ausgeweitet. Ein nicht zu vernachlässigender Teil der Konflikte wurde so auf sehr pragmatische Weise gelöst, ohne dass die strikte Legalität des Urteils dabei zu kontrollieren gewesen wäre. So wurden nahezu 4 Millionen Anliegen (bei einer Bevölkerung von 25 Millionen) jedes Jahr zwischen 1855 und 1871 außerhalb des Gerichtssaals beurteilt - gegenüber 200000 Fällen, die von ,klassischen' Instanzen bearbeitet wurden (Rouet 1998, S. 210).

\subsection{Die Entwicklung des Staates und die Durchsetzung des Rechts}

Diese „Friedensrichter“ blieben dem französischen Rechtssystem bis 1958 erhalten, woraus zu schließen sein könnte, dass bis zu diesem Zeitpunkt eine große Zahl von Streitfällen der öffentlichen Debatte und der juristischen Argumentation entgangen wäre. Tatsächlich setzte sich jedoch das Recht und die ihm eigene Forderung nach Transparenz der Prozesse für quasi die Gesamtheit der Streitigkeiten ab dem Ende des 19. Jh. durch. Eine erste Erklärung könnte darin liegen, dass sich während dieser Zeit der Staat gerade durch den Rekurs auf die Friedensjustiz zunehmend durchsetzt. Der Staat nimmt sich so zahlreicher sozialer Probleme an: der Handhabung der Streiks, der Arbeitsbedingungen, der Betreuung der Armen, etc. Um diese vielfachen Maßnahmen zu implementieren, stützt er sich auf lokaler Ebene auf das Netz der Friedensrichter. 
Diese werden von der Verwaltung damit beauftragt, Untersuchungen zu führen, in Berufsverhandlungen einzugreifen oder Straffällige zu sanktionieren. Über seine juristische Rolle hinaus wird der Friedensrichter so in Abwesenheit spezialisierter Institutionen ein lokaler Akteur der staatlichen Politik. Aus diesem Grund wurden die staatlichen Autoritäten den Bedingungen der Rechtsprechung und dem Respekt des Gesetzes gegenüber immer aufmerksamer. So versicherten sie sich ab 1905 bei der Rekrutierung der juristischen Kompetenzen der Richter, und schafften 1915 die bis dahin bestehende Möglichkeit ab, von den Gesetzen abweichende Urteile zu fällen.

Darüber hinaus muss beachtet werden, dass diese Veränderungen auf geringen Widerstand innerhalb des Berufsstandes gestoßen sind. Dies spiegelt sich auch in den beruflichen Fachzeitschriften der Zeit eindeutig wider. Die Friedensrichter, die vormals durch ihre soziale Herkunft über eine traditionelle Autorität bei der lokalen Bevölkerung verfügten, empfanden dann ein zunehmendes Bedürfnis, sich über das Gesetz auf die staatliche Autorität zu berufen. Ein Teil der Friedensrichter, der städtischen und höher gebildeten Ursprungs war, versuchte dann, diese technischen Kompetenzen weiter zu entwickeln, um eine Abwertung des Berufes zu verhindern. Letztere drohte durch zunehmende Schwierigkeiten bei der Rekrutierung, eine Abnahme der Schlichtungsaktivitäten und die öffentliche Infragestellung ihrer beruflichen Aktivitäten. Aus dieser Perspektive ergab sich der Wille des Berufsstandes, der übrigen Magistratur zu folgen und sich der „Wissenschaft des Rechtes" zu unterstellen.

Dies führte den Berufsstand dazu, ab 1906 über seine repräsentativen Instanzen die Einführung einer beruflichen Eignungsprüfung zu fordern ${ }^{2}$, und in den zwanziger Jahren sogar seine eigene Integration in die gewöhnliche Richterschaft zu verlangen ${ }^{3}$. Diese Entwicklung wurde auch durch eine tief greifende Veränderung der Beziehungen zu den der Gerichtsbarkeit Unterworfenen geprägt, da die Friedensrichter nun über verschiedene Mittel in ihren Gerichten Distanz und die Respektierung der Formalitäten, wie sie in anderen übergeordneten juristischen Institutionen üblich war, einführten. Hierzu zählten beispielsweise die Öffentlichkeit der Verhandlungen, die Anhörungen ausschließlich im Gerichtssaal oder die Neutralität des Richters.

Die Justiz, vor allem bezüglich der Rechtsprechung in kleineren Fällen, scheint also von der Entwicklung der staatlichen Macht beeinflusst zu sein. Die Zunahme der sozialen Gesetzgebung einerseits und die Zentralisierung der politischen Macht andererseits zwingen die ,kleinen' Richter wie auch insbesondere die ihrer Rechtsprechung Unterworfenen dazu, objektivere und strengere juristische Verfahrensweisen anzuwenden. Von dieser Zeit an wurden die informellen Einigungen und Verhandlungen seltener und die ehemals gültige Unterscheidung zwischen großen und kleinen Konflikten hatte keinen Sinn mehr. 1958 wird diese endgültig zusammen mit der Funktion der Friedensrichter abgeschafft.

La justice de paix. Organe des juges de paix, de leurs suppléants, ..., 19, 10. Jan. 1906, S. 1.

Das Handlungsprogramm der „Union Amicale“, Justice de paix, 354, Jan. 1926, S. 2. 


\section{Die Zweideutigkeiten einer Liberalisierung des Rechtes am Beispiel der „bür- gernahen Justiz“}

\subsection{Rückblick auf die Entstebung einer büngernaben Justiz.}

Der bisher entwickelte historische Überblick dient dem besseren Verständnis der aktuellen Transformationen. Das Rechtsmodell, welches 1958 durch die gaullistische Führung durchgesetzt wurde, die selbst stark zentralisiert und technokratisch war, sollte den Anforderungen eines modernen Staates entsprechen und wurde nicht in Frage gestellt. Noch in den 1990er Jahren schien die Idee des stark homogenisierten Gerichtswesens eindeutig: Professionelle Richter wurden mittels eines Auswahlverfahrens eingestellt, in einer spezialisierten Berufsschule („École Nationale de la Magistrature") ausgebildet und regelten alle Streitigkeiten innerhalb der sog. ,Justizpaläste", den Gerichten. Nur einige wenige juristische Aktivitäten wurden aus Sicht der Institution als nur am Rande bedeutend beurteilt und wichen daher von dem Modell der im Straf- und im bürgerlichen Recht praktizierten Vorgehensweise ab: die Streitigkeiten um Arbeitsverträge, bei denen Gewerkschafts- und Arbeitgebervertreter intervenierten, Handelsstreitigkeiten, die durch Mitglieder des Berufsstandes beurteilt wurden, oder auch die Minderjährigenjustiz, die dem Ziel des Schutzes der Kindheit diente.

Gleichwohl setzt Frankreich wie auch andere westliche Länder alternative Formen der Streitschlichtung nach und nach durch. Doch folgt diese Veränderung einer spezifischen Eigenheit des Landes. Über lange Jahre hinweg vermeidet das Land eine ernsthafte theoretische Diskussion über den Stellenwert dieser neuen Handlungsformen in der Rechtsinstitution. Diese bringen eher eine bessere Effizienz mit ein, als dass sie mit den Aktivitäten der Richter konkurrierten. Wenngleich die ersten Erfahrungen bezüglich der Diversifizierung der Bestrafung von Kriminalität oder der Ausübung der Justiz in weniger offiziellen Örtlichkeiten in den 1980er Jahren gemacht wurden, so weisen sie doch die Besonderheit auf, sich in den Kontext von Politiken der Stadtentwicklung einzuschreiben. Die Veränderungen bleiben auf den Randbereich der Arbeit der Justiz beschränkt und sind als einfache Erweiterung der Rolle des Richters konzipiert, die der Staat stärker in die Regulierung bestimmter Stadtviertel einbeziehen möchte. Auch bleibt diese alternative Justiz an spezifische lokale Einzelinitiativen derjenigen Richter gebunden, die sich in dieser neuen sozialen Arbeit betätigen möchten: Sie vertrauen Streitschlichtung lokalen Vereinen an oder beteiligen sich an Präventionsmaßnahmen.

Diese ursprünglichen Bindungen zur Stadtpolitik erklären die spezifische französische Interpretation der „bürgernahen Justiz“. Diese Nähe ist zuerst geographisch, aber ihre Bedeutung verändert sich im Laufe der Zeit mit der Entstehung der alternativen Streitschlichtungsmethoden (Hammouche 1998). Ab Anfang der 1990er Jahre wird dieser Begriff im Allgemeinen gebraucht, um verschiedene Initiativen $\mathrm{zu}$ bezeichnen, die an einigen Gerichten entstanden sind: die Schlichtung, die zwar 1978 beschlossen, aber erst fünfzehn Jahre später wirklich umgesetzt wurde, die Mediation in Strafsachen, die alternativen Prozeduren zur Strafverfolgung (Entschädigungen, zur Ordnung rufen, usw.). Auf der Ebene des Staates werden jedoch keine entscheidenden Veränderungen festgestellt. Zum einen ist die tatsächliche Aktivität dieser Maßnahmen bisher wenig umfangreich (Moreau, Munoz Perez, Serverin 2002), zum anderen bleiben diese Innovationen das Werk der klassischen Justiz: Sie entstehen aus der Initiative beruflicher Richter, die ihre Betätigung ausweiten, indem sie juristische Praktiken entwickeln (Mediation, Schlichtung), die eher als komplementär denn als alternativ wahrgenommen werden (Wyvekens 2001). 
Trotzdem verstärkt sich das Phänomen in dem Maße, wie sich die Krise der Justizinstitution verschlimmert. Immer öfter werden die alternativen Streitschlichtungsmethoden nicht mehr als Anpassung der Rechtsinstitution an die Realität vor Ort betrachtet, sondern als Antwort auf die strukturellen Defizite der Gerichte: Komplexität der Prozeduren, Dauer, Kälte, Unangepasstheit an die Streitigkeiten der, einfachen' Bürger. Um die bürgernahe Justiz herum bauen sich so seit Ende der 1990er Jahre zwei Diskurse auf, die Reformen fordern. Einerseits wird ein verbesserter Zugang zur Justiz gefordert, die den Bedürfnissen der Bürger besser entsprechen solle, indem sie das ,juristische Angebot ${ }^{6}$ vergrößert (Pluralität der Beteiligten, Prozeduren je nach Wichtigkeit der Angelegenheit modifizierbar). Andererseits wird ein verbesserter Zugang zum Gesetz gefordert, dem durch die Verbreitung von Informationen (freiwillige juristische Beratung) und eine juristische Begleitung der dem Gesetz Unterworfenen (höhere Entschädigung der Anwaltsgebühren) entsprochen wird. Diese beiden Orientierungen widersprechen sich teilweise. Wenn sie auch beide ein besseres Funktionieren der Gerichtsbarkeit anstreben, so möchte die eine dies mittels einer Verbesserung des Angebots erreichen, die andere dagegen die Nachfrage unterstützen.

\subsection{Analyse despolitischen Projekts}

Aus dieser Perspektive heraus stellt die Schaffung der bürgernahen Richter, die während einer Reform 2002 beschlossen wurde, einen Bruch dar in der Art und Weise, wie in Frankreich alternative Streitschlichtung angegangen wird. Sie schafft im Straf- und Zivilrecht eine neue konkurrierende Rechtsordnung, die das bisherige Rechtsmodell in Frage stellt. Diesbezüglich ist die bürgernahe Justiz nun institutionell verwirklicht, auch wenn deren juristische Konstruktion bisher - aber dies ist unvermeidlich - nicht der Gesamtheit aller bisher diesem Terminus untergeordneten Maßnahmen entspricht. Indem sie den Akzent auf die Schlichtung legt, ist diese von nicht professionellen Richtern ausgeübte juristische Instanz damit beauftragt, private Streitigkeiten unterhalb der Streitwertgrenze von $1500 € \mathrm{zu}$ regeln. ${ }^{4}$ Im Februar 2005 wurde diese Kompetenz maßgeblich erweitert, indem sie auch auf juristische Personen bezogen wurde und bis zu einer Summe von $4000 €$ angewandt wird. 5

Zum ersten Mal regelt in Frankreich nun ein Gesetz das Verhältnis zwischen der klassischen und der alternativen Justiz. Es geht nicht mehr nur um vereinzelte lokale Initiativen, sondern um eine geplante Reform des Justizwesens. Es handelt sich um die Entscheidung der öffentlichen Hand, die die Ausübung der Justiz erneuern und an bestimmte soziale Veränderungen anpassen soll. Die heutige Infragestellung der als zu juristisch und zu streng bezeichneten Rechtsprechung und die Tendenz in Richtung der „Nähe“ zu den Bürgern können untersucht werden. Dies ist unmittelbar mit einer Reflexion über die Rolle des Staates verbunden: Wie kann dessen Rolle in der juristischen Sphäre beschränkt werden?

4 Die Gesetze vom 10.09.2002 (http://www.legifrance.gouv.fr/citoyen/jorf_nor.ow?numjo=JUSX0200117L) und vom 27.02.2003 (http://www.legifrance.gouv.fr/WAspad/UnTexteDeJorf?numjo $=$ JUSX0200121L).

5 Das Gesetz vom 27.01.2005 (http://www.legifrance.gouv.fr/citoyen/jorf_nor.ow?numjo=JUSX0407800L). 
Wenn man dem Gedankengang der französischen Parlamentarier bei der Einführung der neuen Gerichtsbarkeit (der Justice de proximitê) 2002 folgt (Haenel, Arthuis 1992, Fauchon 1996, Cointat 2001, Fauchon 2002), wird deutlich, dass das Hauptanliegen, welches die Entscheidung zugunsten der „bürgernahen Justiz“ beeinflusst hat, das des mit der Anzahl der Anliegen völlig überforderten Justizapparates ist. ${ }^{6}$ Obwohl die Zahl der Streitfälle seit dem Ende des 19. Jahrhunderts kontinuierlich abgenommen hatte, ist die französische Justiz seit den 1970er Jahren von einer massiven Zunahme der vor Gericht zu verhandelnden Streitigkeiten geprägt. Zunächst als positives Zeichen der „Zivilisierung" empfunden, führte diese Zunahme nach und nach zu einem Problem für das Gerichtswesen, das trotz der signifikanten Aufstockung seiner Mittel außer Stande war, angemessene Fristen bis zur Urteilsverkündung einzuhalten.

Ab Anfang der 1990er Jahre wurde die häufigere Anrufung der Gerichtsbarkeit schließlich als reales Problem oder sogar als krankhaft erkannt. Bis auf die Ausnahmen wirklicher Fallstudien wird dieses Phänomen politisch als Symptom einer nichtfunktionierenden Gesellschaft dargestellt, da die letzte Instanz, der sich Individuen für die Beilegung ihrer Streitigkeiten zuwendeten, die Justiz sei. In dieser Hinsicht könne eine gewisse Anzahl an Streitigkeiten auf einer Ebene unterhalb der eigentlich juristischen verhandelt werden. Für die Politiker erscheint es als kontraproduktiv, die Mittel der Justiz und des Rechtes zu mobilisieren, um diese Angelegenheiten zu lösen. Diese Analysen stimmen mit der Darstellung der Richter überein, die das Recht für unangemessen zur Beurteilung bestimmter Anliegen, die ihnen präsentiert wurden, hielten, und sich auf andere Formen der Konfliktbeilegung beriefen.

Politisch ist die Schaffung der ,bürgernahen Richter" so in Frankreich als Mittel einer Wiederausrichtung der Justiz auf ihre hoheitlichen Funktionen empfunden worden, die ihre Handlung auf bestimmte Angelegenheiten der Gesellschaft beschränkt (Jacob, Blankenburg, Kritzer, Provine, Sanders 1996). So kommt auch die Debatte über das Öffentliche und das Private durch die Differenzierung zwischen verschiedenen Arten der Streitigkeiten wieder auf. Im französischen Diskurs spricht man so einerseits von ,komplexen Streitfällen" (contentieux complexes), deren Regelung sich auf das Recht berufen müssten, und andererseits von „,bürgernahen Streitigkeiten“ (contentieux de proximité), deren Beilegung keine Rechtsprechung sondern eine Schlichtung erfordere. Abgesehen von einer etwaigen empirischen Überprüfung begründen die Verfechter der Justizreform die Explosion der Anzahl der gerichtlichen Verfahren mit der Zunahme der Fälle der zweiten Kategorie von Streitigkeiten. Gewisse soziale Konflikte werden so verdächtigt, zu viel Öffentlichkeit zu erhalten, oder zumindest von den Gerichten eine unmögliche Lösung zu verlangen.

Die Neuausrichtung der Rolle des Staates in der Justiz wird auch durch die Bestrebungen der Parlamentarier bestätigt, alternative soziale Autoritäten zu finden, die dazu fähig sind, diese Art der Regulierung auszuüben: Wer kann heute in der Gesellschaft die Beilegung kleinerer Streitigkeiten übernehmen? Die Debatten der Parlamentarier betreffen dementsprechend hauptsächlich die Wahl dieser neuen Akteure der Justiz, bei der zwei schwierige Bedingungen zu erfüllen seien: Einerseits einen genügend großen Pool zur Rekrutierung von Einzelpersonen, die sich zu dieser Tätigkeit „,berufen“ fühlten, aufzustellen, andererseits hierbei denjenigen gesellschaftlichen Schichten Vorrang einzuräu-

6 Die Entwicklungen der hier dargestellten Überlegungen stützen sich im Wesentlichen auf die Analyse der parlamentarischen Berichte der obersten Kammer (des „Senats"). Es sind in der Mehrzahl französische Senatoren, die sich um eine Reform des Justizwesens bemühen. 
men, die über genügend Autorität verfügen, um dem neuen Gericht Gewicht zu verleihen. Die letztendlich gefundene Lösung ist sehr ambitiös, da sie die Pforten der Justiz außer für die formal hierfür Qualifizierten auch für alle Einzelpersonen öffnet, die in ihrer bisherigen Berufserfahrung als Angestellte in Unternehmen (in Frankreich als "cadres" bezeichnet) Führungspositionen inne hatten.

\subsection{Die Grenzen der Durcbfiubrbarkeit}

Stehen wir heute vor der Wiedereinführung eines Systems, wie es etwa vor der Entwicklung des Zentralstaates Ende des 19. Jahrhunderts existierte? Die Wahrheit ist komplexer. Zunächst muss angemerkt werden, dass das politische Vorhaben, neue Akteure in das juristische System einzuführen, auf den Widerstand der gesamten Richterschaft und auf die Eigenlogik der geteilten beruflichen Normen gestoßen ist. Der Verstoß des Gesetzes gegen das Rechtsstaatsprinzip führte zu der Annullierung von Teilen des vom Parlament angenommenen Gesetzes durch den „Verfassungsrat" (Conseil constitutionnel). Einzig den juristischen Berufen und den juristischen Experten von Betrieben wurde vom Staat die Kompetenz bescheinigt, im Rahmen der „bürgernahen Justiz“ tätig zu sein. Der französische Staat konnte sich also nicht einfach bestimmter juristischer Aufgaben entledigen. Wenn der Formalismus der juristischen Debatten und Urteile zwar umstritten ist, so scheint er heute jedoch gleichzeitig unumgänglich. Auch die „bürgernahen Richter" (Juges de proximite) sind so verpflichtet, innerhalb eines Tribunals zu agieren und die Tatsachen im Lichte des Rechts zu betrachten.

Ganz im Gegensatz zu den politisch vorgegebenen Zielen bedeuten diese also nicht etwa einen Rückzug auf das Private. Die an einigen „bürgernahen Tribunalen“ (Justice de proximitê) durchgeführten Beobachtungen zeigen zwar einen oft unzufriedenen Richter, da dieser gleichzeitig mit schweren juristischen Unzulänglichkeiten (fehlende Beweise, schlechte juristische Beschreibung der Tatsachen) konfrontiert wird. Durch das Gesetz gebunden muss der „,bürgernahe Richter“ nur allzu oft die Anfragen der Bürger ablehnen. In der Realität beschränkt sich die angestrebte „Nähe“ der Justiz darauf, öffentlich die Arbeit eines Rechtsanwaltes zu erledigen, also auf die juristische Aufbereitung des Anliegens.

Es handelt sich bei der heutigen Praxis also um ein anderes Modell der „,bürgernahen Justiz" als das ursprünglich politisch vorgesehene. Tatsächlich geht es vielmehr darum, den Bürgern den Zugang zur Justiz zu erleichtern. Die freien juristischen Berufe (Rechtsanwälte, Gerichtsvollzieher) haben zunehmend die Stellen der „, bürgernahen Richter" inne und stellen so die Gesetze in das Zentrum ihrer Arbeit. Wenn also eine Wiederkehr der Unterscheidung zwischen den Streitigkeiten festzustellen ist, so kann sie jedoch bisher nur auf der Ebene der Akteure (professionelle/nicht-professionelle), nicht aber derjenigen der Justiz an sich ausgemacht werden.

Nach weniger als dreijähriger Implementierung hat diese neue Jurisdiktion wesentliche Veränderungen durchlaufen, die die aktuellen Instanzen in hohem Maße von dem ursprünglich von den Senatoren und Abgeordneten gedachten Modell abweichen lassen. Fast die Gesamtheit der Eigenschaften, die die bürgernahen Richter der alternativen Streitschlichtung annäherten, sind heute aufgehoben worden: Eine Urteilsverkündung wird weitgehend einer Schlichtung vorgezogen, die Möglichkeiten, außerhalb der Gerichte zu urteilen sind tatsächlich nie verwirklicht, die nicht-professionellen Richter sind qualifizierte Juristen und die Angelegenheiten werden gemäß dem geltenden Recht entschieden. 
Wenn auch die politische Ausarbeitung der Reform durch die Exekutive und die Legislative zeigt, dass die Idee eines Rückzugs des Staates in den Reformprojekten der Rechtsinstitution präsent ist, muss deren tatsächliche Verwirklichung jedoch mit Vorsicht beurteilt werden. Noch heute macht es die Situation der französischen Richterschaft schwer, einen Teil der sozialen Pflicht der Justiz aufzugeben zugunsten der alternativen Praktiken des Rechts.

Dieser Beitrag zeigt also auf, wie empfindlich die Justiz in Frankreich auf die Regulierung durch den Staat reagiert. Während seiner Entstehung in den letzten zwei Jahrhunderten hat die dem Gesetz zugewiesene Schlüsselfunktion dazu geführt, dass der Richter zum einfachen „Weberischen Funktionär" wurde: Neutral, in jeder seiner Handlungen objektiv arbeitend und auf eine klare Darlegung der Tatsachen bedacht. Das Gerichtswesen, und innerhalb dessen die professionellen Richter, haben ergo in der gesellschaftlichen Regulierung eine herausragende Rolle eingenommen, während andere Akteure, die oftmals am Rande dieser Institution oder auf der Ebene unterhalb der Gerichtsbarkeit involviert waren, an Bedeutung verloren haben und verschwunden sind. Heute hat die Infragestellung des jakobinischen Staates (Duran 1993) zwangsläufig Auswirkungen auf die Organisation der Justiz. Der Grundsatz, alles dem Juristischen zuzuordnen, scheint in Zweifel gezogen zu werden, und zwar zugunsten einer neuen Aufteilung der juristischen Kompetenzen zwischen verschiedenen gesellschaftlichen Akteuren. In diesem Sinne ist das Dilemma zwischen das Privatem und das Öffentlichem noch aktuell. Es geht nicht um einen Rationalisierungsprozess (Weber 1921), der das Öffentliche als eine überhaupt bessere Justizform gefördert hätte. Noch heute sind private Formen angemessen, je nach dem welche Rolle der Zentralstaat in der Regulierung der Gesellschaft spielen will.

Gleichzeitig wird aber auch deutlich, dass es trotz des expliziten politischen Willens, zu einem Modell der Justiz zurückzukehren, welches auf außergerichtliche Institutionen und Verhandlungen beruht, nicht dazu kam. Falls derartige Institutionen heute bestehen (wie die Schlichtung oder die Mediation), so nur am Rande des juristischen Apparates und zu dem Zweck, das Gesetz in bestimmten Situationen, weicher' anzuwenden. Doch innerhalb des bestehenden Justizapparates scheint es heute schwierig, eine regelrechte soziale Autorität zu etablieren, die Konflikte schlichten und Verhandlungen begünstigen könnte. Das hier aufgegriffene Beispiel der „,bürgernahen Richter“, wo es dem sich zurückziehenden Staat nicht gelang, eine Justiz fernab der öffentlichen Aufmerksamkeit zu schaffen, zeigt auch die Stärke des Rechtes, welches sich von nun an über die staatliche Autorität stellt.

Angesichts der aktuellen Schwierigkeiten der Richter ist es für einige Abgeordnete des französischen Parlaments um so einfacher, neue Optionen für den differenzierten Umgang mit juristischen Angelegenheiten vorzuschlagen, der nun von der jeweiligen Bedeutung der Vergehen abhängt. Diese neuen Akteure als „Richter“ zu bezeichnen, widerspricht jedoch dem System selbst, welches sich auf den professionellen Richterstand stützt. Diese Stütze ist eben gerade auf diese Weise konzipiert, um das System vor in verschiedenen Phasen seiner Geschichte erfahrenem politischem Druck zu bewahren. Nicht nur der etablierte Berufsstand der Richter wehrt sich gegen die „,bürgernahen Richter“. Die obersten Gerichte zögern vor dieser Reform und beschränken sie. Die Europäische Menschenrechtskonvention sowie die verfassungsrechtliche Erklärung der Menschenund Bürgerrechte von 1789 werten die gewählte Lösung ab, indem sie die Charakteristika eines fairen Verfahrens darlegen. Die Experten des Rechtswesens sorgen sich um die Zwei-Klassen-Gerechtigkeit. Für zahlreiche in diesem Feld einflussreiche Persönlichkei- 
ten konnte die Einführung der bürgernahen Justiz also nur innerhalb des existierenden legalen Rahmens durchgesetzt werden. Die historische Konstruktion des Richtertums ist sowohl bei den jeweiligen Akteuren als auch in deren Handlungslogik fest verankert. Dies in Frage zu stellen, würde auf der politische Ebene die Akzeptanz einer Diversifizierung des Status' der Richter an sich bedeuten. Diese grundlegende Veränderung wird derzeit jedoch noch nicht explizit thematisiert.

Literatur

Arthuis, Jean, Haenel, Hubert (1992): Justice sinistrée, démocratie en danger, Paris, Economica.

Bonafé-Schmitt, Jean-Pierre (1987): „La part et le rôle joués par les modes informels de règlement des litiges dans le développement d'un pluralisme judiciaire (Etude comparative FranceUSA)“, In: Droit et Société, 6, S. 253-275.

Bonafé-Schmitt, Jean-Pierre (2005): „La médiation : une nouvelle forme d'action“. In: Faget, Jacques (Hrsg.), Médiation et action publique - La dynamique du fluide, Pessac, Presse universitaire de Bordeaux, S. 63-87.

Cointat, Christian (2002): Rapport d'information sur l'évolution des métiers de la justice, Paris, Sénat, $\mathrm{N}^{\circ} 345$. http://www.senat.fr/rap/r01-345/r01-345.html

Commaille, Jacques (1999): „La déstabilisation des territoires de justice“. In: Droit et Société, 42/43, S. 239-264.

Cottereau, Alain (2002): „Droit et bon droit - Un droit des ouvriers instauré, puis évincé par le droit du travail (France, XIXe siècle) ${ }^{“ .}$. In: Annales HSS, No 6 , S. 1521-1557.

Duran, Patrice (1993): „Piloter l'action publique, avec ou sans droit ?“. In: Politiques et management public, Band 11, 4 .

Fauchon, Pierre (1996): Quels moyens pour quelle justice?, Paris, Sénat, N49. http://www.senat.fr/rap/r96-49/r96-49.html

Fauchon, Pierre (2002): Rapport fait sur le projet de loi organique relatif aux juges de proximité, Paris, Sénat, $\mathrm{N}^{\circ}$ 404. http://www.senat.fr/rap/101-404/101-404.html

Giraud, Claude (1993): L'action commune - essai sur les dynamiques organisationnelles, Paris, l'Harmattan.

Glick, Henry R. (1983): Courts, Politics and Justice, New York, McGraw-Hill.

Hammouche, Abdelhafid (1998): „La politique de la ville entre médiation et proximitéc, In: Droit et Société, 38, S. 109-129.

Harrington, Christine B. (1988): „Regulatory Reform : Creating Gaps and Making Markets“. In: Law ¿r Policy, Band 10, 4, Oktober.

Jacob, Herbert, Blankenburg, Erhard, Kritzer, Herbert M., Provine, Doris Marie, Sanders, Joseph (1996): Courts, Law \& Politics in Comparative Perspective, New Heaven, London, Yale University Press.

Le Roy, Ėtienne (1995): „La médiation, mode d’emploi“, In: Droit et Société, 29, s. 39-55.

Moreau, Caroline, Munoz Perez, Brigitte, Serverin, Évelyne (2002): La médiation judiciaire civile en chiffres, Paris, Ministère de la Justice. http://lesrapports.ladocumentationfrancaise.fr/cgi$\mathrm{bin} / \mathrm{brp} /$ telestats.cgi?brp_ref $=044000378 \& \mathrm{brp} \_$file $=0000$.pdf

Rouet, Gilles (1998): „La justice de paix en France : une expérience de la conciliation“, In: Chauvaud, Frédéric, Petit, Jacques-Guy, L'bistoire contemporaine et les usages des archives judiciaires (18001939), coll. "Histoire et Archives", Paris, Honoré Champion Editeur, S. 205-226.

Weber, Max (1921): Wirtschaft und Gesellschaft, Tübingen, Mohr-Siebeck.

Wyvekens, Anne (2001): „La justice de proximité en France : politique judiciaire de la ville et interrogations sur la fonction de justice", In: Faget, Jacques, Wyvekens, Anne (Hrsg.), La justice de proximité en Europe - Pratiques et enjeux, Coll. "Trajets", Ramonville Saint-Agne, Erès, S. 17-36. 